\title{
THE MEAN CONVERGENCE OF ORTHOGONAL SERIES. I
}

\author{
BY \\ HARRY POLLARD( $\left.{ }^{1}\right)$
}

1. Introduction. Let $\phi_{n}(x), n=0,1, \cdots$, be a set of functions orthonormal on an interval $(a, b)$, and belonging to both a Lebesgue space $L^{p}(a, b)$ and its conjugate $L^{p^{\prime}}(a, b), p>1$. We shall say that the set has the property of $p$ mean convergence or forms a basis for $L^{p}(a, b)$ if the formal expansion

$$
f(x) \sim \sum_{0}^{\infty} \phi_{n}(x) \int_{a}^{b} f(y) \phi_{n}(y) d y
$$

of every function $f(x)$ in $L^{p}(a, b)$ converges to $f(x)$ in the pth mean.

A classical theorem of Marcel Riesz [1]( $\left.{ }^{2}\right)$ asserts that the functions $(2 / \pi)^{1 / 2} \cos n x, n=0,1, \cdots$, form a basis for $L^{p}(0, \pi)$ for all $p>1$. An examination of the literature discloses only three further sets for which the property has been studied $\left({ }^{3}\right)$ : the Haar functions [2], the Fourier transforms of the Laguerre polynomials $[3,4]$, and the Walsh functions [14]. These sets are bases for $L^{p}(0,1), L^{p}(-\infty, \infty)$, and $L^{p}(0,1)$ respectively, for all values of $p>1$ (in the case of the Haar functions for $p=1$ also).

It is the object of this study to investigate the extent to which the property of $p$-mean convergence is shared by other sets of functions. The methods of functional analysis supply useful necessary and sufficient conditions, but there seems to be no general method for determining whether a given set actually fulfills them, and each must be examined on its own merits.

It is natural to begin with a set of functions as nearly like $\{\cos n x\}$ as possible. The particular choice made here is the set $\left\{\cos \alpha_{n} x\right\}$ arising from a simple Sturm-Liouville problem

$$
\begin{gathered}
y^{\prime \prime}+\alpha^{2} y=0 ; \quad K y(\pi)+y^{\prime}(\pi)=0, \\
y^{\prime}(0)=0 ; \quad K>0
\end{gathered}
$$

on the interval $(0, \pi)$. Since $\alpha_{n}=n+O(1 / n)$ it is reasonable to expect that the set will behave with respect to mean convergence exactly like the set $\{\cos n x\}$. This we verify in Chapter I. The famous integral inequality of Hilbert [6;

Presented to the Society, April 27, 1946; received by the editors November 7, 1946.

(1) The theorems of Chapter II were obtained in 1945 when the author was a Jewett Fellow at Yale University.

(2) Numbers in brackets refer to the bibliography at the end of the paper.

(8) This does not include results for Legendre and other orthogonal polynomials announced recently by the author without proof [5]. The missing proofs will be supplied in this paper and a sequel. 
226] turns up in unexpected fashion as the crux of the proof.

That the result remains valid when $\{\cos n x\}$ is replaced by more general Sturm-Liouville sets is a simple consequence of the equiconvergence theory of Sturm-Liouville expansions. From this point of view our proof that $\left\{\cos \alpha_{n} x\right\}$ forms a basis for $L^{p}(0, \pi), p>1$, is needlessly complicated. Nevertheless our proof can be carried over to sets of functions for which the equiconvergence method fails (for example, the functions considered in Chapter III), and for this reason we have considered it worthwhile to present it here.

When the set is chosen to be the normalized Legendre polynomials on $(-1,1)$ the situation alters. A simple example $(\$ 7)$ already shows that the property of mean convergence fails for these functions if $1<p<4 / 3$; it follows by a general principle (\$2) that it must also fail for the conjugate exponents $p>4$. Chapter II is devoted to a proof of Zygmund's (unpublished) conjecture that the property does hold for $4 / 3<p<4$. Here again the proof eventually reduces to an integral inequality $(\$ 12)$. This inequality appears to be new, and may be of some interest in itself. The status of the end-values $p=4 / 3, p=4$ is open; analogy with the corresponding situation (which occurs at $p=1, p=\infty)$ for trigonometric series suggests failure of the prcperty at these critical values, but I am unable to confirm this.

Paralleling the step taken in generalizing the functions $\{\cos n x\}$ to $\{\cos \alpha, x\}$, we consider finally Hille's crthogonal sets of spherical harmonics $\left\{P_{\alpha_{n}}(x)\right\}$, where the $\alpha_{n}$ are no longer integers [7]. It is not surprising that the results turn out to be the same as for Legendre series (Chapter III).

2. General principles. Before proceeding to the specific problems it is convenient to state some general principles. It is assumed that $\left\{\phi_{n}(x)\right\}$ is orthonormal, belongs to $L^{p}(a, b)$ and $L^{p^{\prime}}(a, b)$, and is closed in both spaces $\left(^{4}\right)$. As usual, $\|f\|_{p}$ denotes the norm $\left(\int_{a}^{b}|f(x)|^{p} d x\right)^{1 / p}$ of an element $f(x)$ of $L^{p}(a, b)$. $S_{n}(f)$ or $S_{n}(f ; x)$ will be used for the partial sums of the expansion of $f(x)$. Then:

(i) If $\left\{\phi_{n}\right\}$ is a basis for $L^{p}(a, b), p>1$, it is also a basis for $L^{p^{\prime}}(a, b)$.

Obviously the failure of the property for a value of $p$ implies its failure for $p^{\prime}$. Furthermore, we may limit our attention to the interval $1<p \leqq 2$; this is particularly convenient in obtaining negative results. The principle (i) can be found in Banach $[8 ; 108]$ as a corollary of more general results.

(ii) To establish that $\left\{\phi_{n}(x)\right\}$ is a basis for $L^{p}(a, b), p>1$, it is sufficient to prove that, for all elements $f(x)$ of $L^{p}(a, b)$,

$$
\limsup _{n \rightarrow \infty}\left\|S_{n}(f)\right\|_{p}<\infty .
$$

We point out that (2.1) is sufficient only in the presence of the hypothesis of closure assumed at the beginning of the section. To establish (ii) observe first that $\left\{S_{n}(f)\right\}$ is a sequence of linear transformations of $L^{p}$. From (2.1)

(4) A set is closed if its closed linear span is the entire space. 
and a theorem of Banach and Steinhaus $[8 ;$ p. 80, Théorème 5] it follows that $\left\|S_{n}(f)\right\|_{p} \leqq A_{p}\|f\|_{p}$, where $A_{p}$ is independent of $n$ and $f$. Now, since the $\phi_{n}(x)$ are closed, we may invoke another one of their theorems [ $8 ; p .79$, Theorème 3 ] which implies in this case that $1 . \mathrm{i} . \mathrm{m} \cdot{ }_{n \rightarrow \infty}^{(p)} S_{n}(f ; x)$ exists for all $f(x)$. It remains only to identify this mean limit-call it $g(x)$-with $f(x)$. Since

$$
\lim _{n \rightarrow \infty} \int_{a}^{b}\left|S_{n}(f ; x)-g(x)\right|^{p} d x=0
$$

then

$$
\lim _{n \rightarrow \infty} \int_{a}^{b} \phi_{m}(x)\left(S_{n}(f ; x)-g(x)\right) d x=0
$$

for all $m$. From the orthonormality of the $\phi_{m}(x)$ we can conclude that

$$
\int_{a}^{b}[f(x)-g(x)] \phi_{m}(x) d x=0
$$

for all $m$. Hence $f(x) \equiv g(x)$.

\section{Chapter I. Generalized trigonometric Functions}

3. The functions $\left\{\cos \alpha_{n} x\right\}$. From the equations (1.1) it follows that the $\alpha_{n}$ are the positive roots of the equation

$$
\alpha \sin \alpha \pi=K \cos \alpha \pi \text {. }
$$

These roots are the abscissas of the intersections of the two curves $y=K / \alpha$, $y=\tan \alpha \pi$ in the first quadrant. Consequently $\alpha_{n}=n+\epsilon_{n}, n=0,1, \cdots$, $0<\epsilon_{n}<1 / 2$. But by (3.1)

$$
\tan \epsilon_{n} \pi=K / \epsilon_{n}+n=O(1 / n),
$$

so that $\epsilon_{n}=O(1 / n)$. Hence $\alpha_{n}=n+O(1 / n)$. It follows from theorems of Levinson $\left[9 ;\right.$ pp. 6-7] that the functions $\left\{\cos \alpha_{n} x\right\}$ are closed $L^{p}(0, \pi)$ for all $p>1$.

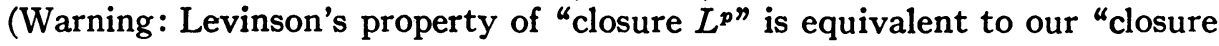
$L^{p^{\prime}} . "$ ) The orthogonality of the set can be verified by direct computation, but already follows from the fact that the functions are a Sturm-Liouville set. The functions $\left\{\sigma_{n}^{-1} \cos \alpha_{n} x\right\}$ are orthonormal if

$$
\sigma_{n}^{2}=\int_{0}^{\pi} \cos ^{2} \alpha_{n} x d x=\frac{\pi}{2}+\frac{\sin \alpha_{n} \pi \cos \alpha_{n} \pi}{2 \alpha_{n}} .
$$

THeOREM 3.1. The functions $\left\{\sigma_{n}^{-1} \cos \alpha_{n} x\right\}$ form a basis for $L^{p}(0, \pi)$ for all $p>1$.

In view of the closure of the functions in question, it is sufficient to verify (2.1). This is the object of the following sections. 
4. The functions $\{\cos (n+1 / 2) x\}$. As a first step we show that the orthonormal functions $\left\{(2 / \pi)^{1 / 2} \cos (n+1 / 2) x\right\}_{0}^{\infty}$ are a basis of $L^{p}(0, \pi)$ for all $p>1$.

Suppose $f(x) \in L^{p}(0, \pi)$; extend it to be even on $(-\pi, \pi)$. Then $e^{-i x / 2} f(x)$ $\in L^{p}(-\pi, \pi)$, so that on $(-\pi, \pi)$

$$
e^{-i x / 2} f(x)=\underset{N \rightarrow \infty}{\text {.i.m. }} . \frac{1}{2 \pi} \sum_{-N-1}^{N} e^{i n x} \int_{-\pi}^{\pi} e^{-i(n+1 / 2) y} f(y) d y .
$$

This is a consequence of Riesz's theorem $[1],[10 ; 153]$. Since $\left|e^{-i x / 2}\right|=1$ and $f(x)$ is even,

$$
f(x)=\operatorname{lilm}_{N \rightarrow \infty}^{(p)} . \frac{1}{\pi} \sum_{-N-1}^{N} e^{i(n+1 / 2) x} \int_{0}^{\pi} \cos (n+1 / 2) y f(y) d y
$$

on $(-\pi, \pi)$; and

$$
f(x)=\operatorname{lil}_{N \rightarrow \infty}^{(p)} . \frac{2}{\pi} \sum_{0}^{N} \cos (n+1 / 2) x \int_{0}^{\pi} \cos (n+1 / 2) y f(y) d y
$$

on $(0, \pi)$. This completes the proof.

Let $\sum_{n}(f)$ denote the partial sums of the expansion of $f(x)$ in the set $\{\cos (n+1 / 2) x\}$. Then we have established in particular that

$$
\lim _{n \rightarrow \infty}\left\|\sum_{n}(f)\right\|_{p}=\|f\|_{p}
$$

5. A contour integral. Let $K$ be the fixed positive constant of (3.1), and suppose $0<s<\pi, 0<t<\pi$. Choose $R_{N}$, so that $\alpha_{N+1}>R_{N}>N+1 / 2>\alpha_{N}$ and let $C(N)$ be the contour which consists of the line segment joining $i R_{N}$ to $-i R_{N}$, together with the semicircle which has the segment as diameter and lies in the right half-plane. Define the integral $I$ by

$$
I=\frac{1}{2 \pi i} \int_{C(N)} \frac{2 z \cos z s \cos z t d z}{(z \sin \pi z-K \cos \pi z) \cos \pi z} .
$$

Let $C_{1}(N)$ be the curved and $C_{2}(N)$ the straight part of the contour. Along $C_{2}(N)$ the integral is

$$
\int_{R_{N}}^{-R_{N}} \frac{2 y \cosh y s \cosh y t d y}{(y \sinh \pi y+K \cosh \pi y) \cosh \pi y} ;
$$

this vanishes since the interval is symmetric in the origin and the integrand is odd. Hence

$$
I=\frac{1}{2 \pi i} \int_{C_{1}(N)} \cdots d z .
$$


Now the singularities of the integrand in (5.1) are simple poles occurring at $\alpha_{k}$ and $k+1 / 2, k=0,1, \cdots, N$. Then by (5.2) and the residue theorem

$$
\sum_{0}^{N} \frac{\cos \alpha_{k} s \cos \alpha_{k} t}{\sigma_{k}^{2}}-\frac{2}{\pi} \sum_{0}^{N} \cos (k+1 / 2) s \cos (k+1 / 2) t
$$

$$
=\frac{1}{2 \pi i} \int_{C_{1}(N)} \cdots d z .
$$

We next obtain an estimate of the right-hand member, independent of $N$.

$$
\begin{aligned}
\left|\frac{1}{2 \pi i} \int_{C_{1}(N)} \cdots d z\right| & =\left|\frac{2}{2 \pi i} \int_{C_{1}(N)} \frac{\cos z s \cos z t}{\cos z \pi \cos z \pi} \frac{d z}{(\tan z \pi-K / z)}\right| \\
& \leqq A \int_{C_{1}(N)} e^{-(2 \pi-\sigma-t)|y|}|d z| \\
& =A R_{N} \int_{-\pi / 2}^{\pi / 2} \exp \left\{-(2 \pi-s-t) R_{N}|\sin \theta|\right\} d \theta \\
& \leqq A R_{N} \int_{0}^{\pi / 2} \exp \left\{-(2 \pi-s-t) R_{N} \sin \dot{\theta}\right\} d \theta .
\end{aligned}
$$

Now let $a=R_{N}(2 \pi-s-t)$ and make the change of variable $v=a \sin \theta$. The last term becomes

$$
\frac{A}{2 \pi-s-t} \int_{0}^{a} \frac{e^{-v} d v}{\left[1-(v / a)^{2}\right]^{1 / 2}}
$$

and this is in turn less than or equal to

$$
A /(2 \pi-s-t)
$$

From (5.3) we obtain then that

$$
\begin{aligned}
\mid \sum_{0}^{N} \frac{\cos \alpha_{k} s \cos \alpha_{k} t}{\sigma_{k}^{2}}- & \frac{2}{\pi} \sum_{0}^{N} \cos (k+1 / 2) s \cos (k+1 / 2) t \mid \\
& \leqq \frac{A}{2 \pi-s-t},
\end{aligned}
$$

where $A$ is independent of $N, s$, and $t$.

6. Proof of Theorem 3.1. To prove the theorem we have only to verify that the partial sums $S_{n}(f)$ of the expansion of any function $f(x)$ satisfy (2.1). Employing the notation of $\$ 4$, we obtain from (5.4) that

$$
\left|S_{N}(f ; s)-\sum_{N}(f ; s)\right| \leqq A \int_{0}^{\pi} \frac{|f(t)| d t}{2 \pi-s-t} .
$$


Hence

$$
\left\|S_{N}(f)\right\|_{p} \leqq\left\|\sum_{N}(f)\right\|_{p}+A\|\psi(s)\|_{p}
$$

where

$$
\psi(s)=\int_{0}^{\pi} \frac{|f(t)|}{2 \pi-s-t} d t .
$$

In view of (4.1), the condition (2.1) is satisfied if $\psi(s)$ belongs to $L^{p}(0, \pi)$. By the converse of Hölder's inequality the latter fact will be established if we can prove that

$$
J=\int_{0}^{\pi} g(s) d s \int_{0}^{\pi} \frac{|f(t)|}{2 \pi-s-t} d t
$$

exists for every function $g(s)$ of $L^{p^{\prime}}(0, \pi)$. But

$$
J=\int_{0}^{\pi} g(\pi-s) d s \int_{0}^{\pi} \frac{|f(\pi-t)| d t}{s+t},
$$

and the existence of $J$ follows immediately from Hilbert's integral inequality $[6 ;$ p. 226].

\section{Chapter II. The Legendre polynomials}

7. Legendre series for $1<p<4 / 3$. If $f(x) \in L(-1,1)$ it has a formal Legendre series

$$
f(x) \sim \sum_{0}^{\infty} a_{n} P_{n}(x)
$$

where $a_{n}=(n+1 / 2) \int_{-1}^{1} f(x) P_{n}(x) d x$. Let $S_{N}(f)$ denote, as usual, the partial sums. Since

$$
\left|a_{N}\right|\left(\int_{-1}^{1}\left|P_{N}(x)\right|^{p} d x\right)^{1 / p}=\left(\int_{-1}^{1}\left|S_{N}(f ; x)-S_{N-1}(f ; x)\right|^{p} d x\right)^{1 / p},
$$

a necessary condition for the $p$-mean convergence of a Legendre series is that

$$
\lim _{N \rightarrow \infty} a_{N}\left(\int_{-1}^{1}\left|P_{N}(x)\right|^{p} d x\right)^{1 / p}=0 .
$$

We shall prove that there is a function of $L^{p}(-1,1), 1<p<4 / 3$, for which this condition is violated. It follows that the Legendre polynomials are not a basis if $1<p<4 / 3$; nor, by the results of $\$ 2$, can they be a basis if $p>4$.

The function in question is $f(x)=(1-x)^{-3 / 4}$, a standard counterexample for many purposes. It obviously belongs to $L^{p}(-1,1)$ for $1<p<4 / 3$. More- 
over $\left[11 ;\right.$ p. 249] $a_{N}>A N^{1 / 2}(N \rightarrow \infty)$. Now $[11 ;$ p. 168]

$$
\int_{-1}^{1}\left|P_{N}(x)\right| d x>A N^{-1 / 2}
$$

so that by Hölder's inequality

$$
\left.\int_{-1}^{1}\left|P_{N}(x)\right|^{p} d x\right)^{1 / p} \geqq 2^{-1 / p^{\prime}} \int_{-1}^{1}\left|P_{N}(x)\right| d x>A N^{-1 / 2}
$$

It follows that

$$
\liminf _{N \rightarrow \infty} a_{N}\left(\int_{-1}^{1}\left|P_{N}(x)\right|^{p} d x\right)^{1 / p}>0,
$$

so that (7.1) is not fulfilled.

The argument used here is not unlike that which proves the divergence of a series by showing that the general term does not approach zero, and so is not a very delicate one. It is possible that more refined methods are needed to settle the case $p=4 / 3$.

8. Properties of the Legendre polynomials. The goal of this chapter is to establish the following theorem.

THEOREM 8.1. The Legendre polynomials form a basis for $L^{p}(-1,1)$ if $4 / 3<p<4$.

The proof, which is rather delicate, will be accomplished by a series of reductions which culminate in the inequalities of $\$ 12$. In the present section we shall state the required properties of the polynomials; in particular, we derive a new form of Christoffel's identity more suitable for our purposes than the standard one.

First, some inequalities. We shall need the familiar inequality $[11 ;$ p. 160 , Theorem 3.3]

$$
\left(1-x^{2}\right)^{1 / 4}(n+1)^{1 / 2}\left|P_{n}(x)\right| \leqq A .
$$

Now we require an estimate for the difference $P_{n+2}(x)-P_{n}(x)$. Many writers, for example Watson $[12 ;$ p. 315], give the formula

$$
(n+1)^{1 / 2}\left|P_{n}(x)-P_{n+2}(x)\right| \leqq A,
$$

which is adequate for the treatment of behavior interior to the interval $(-1,1)$. It does not, however, take adequate account of the cancellation of the two terms at the end points $x= \pm 1$. It is precisely at the end points where the difficulties in our problem occur, and so the more informative inequality $[11 ;$ p. $167(7.33 .10)]$

$$
\left(1-x^{2}\right)^{-1 / 4}(n+1)^{1 / 2}\left|P_{n}(x)-P_{n+2}(x)\right| \leqq A
$$


will be used. I have been unable to get along with anything weaker.

It is important to observe that, in view of the relation $[11, p .71]$

$$
\frac{1}{2}\left(1-x^{2}\right) P_{n}^{(1,1)}(x)=\frac{n+1}{2 n+3}\left[P_{n}(x)-P_{n+2}(x)\right],
$$

(8.2) can be written

$$
\left|\left(1-x^{2}\right)^{8 / 4}(n+1)^{1 / 2} P_{n}^{(1,1)}(x)\right| \leqq A
$$

which is precisely the analogue of $(8.1)$ for the Jacobi polynomials $P_{n}^{(1,1)}(x)$. It is this observation which suggested to me the extension of Theorem 8.1 to Jacobi polynomials announced in [5]; the proofs will appear in a sequel.

The partial sums of the Legendre series of $f(x)$ are given by the formula $[11 ; 70]$

$$
S_{N}(f ; x)=\int_{-1}^{1} f(y) K_{N}(x, y) d y,
$$

where

$$
K_{N}(x, y)=\frac{N+1}{2} \frac{P_{N+1}(x) P_{N}(y)-P_{N}(x) P_{N+1}(y)}{x-y} .
$$

This form of the kernels, due to Christoffel, will now be recast in another form, valid for $x \neq y$.

We have, after a simple rearrangement,

$$
\begin{aligned}
K_{N}(x, y)+K_{N+1}(x, y)= & \frac{N+1}{2} P_{N+1}(x) \frac{P_{N}(y)-P_{N+2}(y)}{x-y} \\
& +\frac{N+1}{2}\left[P_{N+2}(x)-P_{N}(x)\right] \frac{P_{N+1}(y)}{x-y} \\
& +\frac{1}{2} P_{N+2}(x) \frac{P_{N+1}(y)}{x-y} \\
& -\frac{1}{2} P_{N+1}(x) \frac{P_{N+2}(y)}{x-y} .
\end{aligned}
$$

The last two terms, by (8.4), sum to $(N+2)^{-1} K_{N+1}(x, y)$. Transferring them to the left-hand side yields

$$
\begin{aligned}
K_{N}(x, y)+\left(1-\frac{1}{N+2}\right) K_{N+1}(x, y) & =\frac{N+1}{2} P_{N+1}(x) \frac{P_{N}(y)-P_{N+2}(y)}{x-y} \\
& +\frac{N+1}{2}\left[P_{N+2}(x)-P_{N}(x)\right] \frac{P_{N+1}(y)}{x-y} .
\end{aligned}
$$


But also $K_{N+1}(x, y)=K_{N}(x, y)+(N+3 / 2) P_{N+1}(x) P_{N+1}(y)$, so that the lefthand side is now

$$
\left(2-\frac{1}{N+2}\right) K_{N}(x, y)+\frac{N+1}{N+2} \frac{2 N+3}{2} P_{N+1}(x) P_{N+1}(y) .
$$

Thus we arrive at the fundamental formula

$$
\begin{aligned}
K_{N}(x, y)= & \frac{N+2}{2 N+3} \frac{N+1}{2} P_{N+1}(x) \frac{P_{N}(y)-P_{N+2}(y)}{x-y} \\
& +\frac{N+2}{2 N+3} \frac{N+1}{2}\left[P_{N+2}(x)-P_{N}(x)\right] \frac{P_{N+1}(y)}{x-y} \\
& -\frac{N+1}{2} P_{N+1}(x) P_{N+1}(y) .
\end{aligned}
$$

This form is obviously designed to make use of the inequality (8.2).

9. Estimates of the partial sums. The closure $L^{p}(-1,1)$ of the Legendre polynomials( $(5)$ may be taken for granted, so that our attention centers on the proof that $(2.1)$ holds for $4 / 3<p<4$. Suppose then that $f(x) \in L^{p}(-1,1)$, $4 / 3<p<4$. By the formulas (8.3) and (8.5)

$$
\begin{aligned}
S_{N}(f ; x)= & \frac{N+2}{2 N+3} \frac{N+1}{2} P_{N+1}(x) \int_{-1}^{1} \frac{P_{N}(y)-P_{N+2}(y)}{x-y} f(y) d y \\
& +\frac{N+2}{2 N+3} \frac{N+1}{2}\left[P_{N+2}(x)-P_{N}(x)\right] \int_{-1}^{1} \frac{P_{N+1}(y) f(y) d y}{x-y} . \\
& -\frac{N+1}{2} P_{N+1}(x) \int_{-1}^{1} P_{N+1}(y) f(y) d y .
\end{aligned}
$$

The first two integrals exist almost everywhere as Cauchy principal values $[10 ;$ p. 317$]$. Then by $(8.1)$ and (8.2)

$$
\left|S_{N}(f ; x)\right| \leqq A(N+1)^{1 / 2}\left(1-x^{2}\right)^{-1 / 4}\left|\int_{-1}^{1} \frac{P_{N}(y)-P_{N+2}(y)}{x-y} f(y) d y\right|
$$

$$
\begin{aligned}
& +A(N+1)^{1 / 2}\left(1-x^{2}\right)^{1 / 4}\left|\int_{-1}^{1} \frac{P_{N+1}(y)}{x-y} f(y) d y\right| \\
& +A(N+1)^{1 / 2}\left(1-x^{2}\right)^{-1 / 4}\left|\int_{-1}^{1} P_{N+1}(y) f(y) d y\right| .
\end{aligned}
$$

We shall first dispose of the last of the three terms on the right-hand side of (9.1) by showing that it has a norm bounded independently of $N$. By

(") Or any sequence of polynomials, one of each degree! 
Hölder's inequality and (8.1)

$$
\begin{aligned}
\left|\int_{-1}^{1} P_{N+1}(y) f(y) d y\right| & \leqq\|f\|_{p}\left(\int_{-1}^{1}\left|P_{N+1}(y)\right|^{p^{\prime}} d y\right)^{1 / p^{\prime}} \\
& \leqq A(N+1)^{-1 / 2}\|f\|_{p}\left(\int_{-1}^{1}\left(1-y^{2}\right)^{-p^{\prime} / 4} d y\right)^{1 / p^{\prime}},
\end{aligned}
$$

which is finite since $p^{\prime}<4$. Then the third term in question is less than or equal to

$$
A\|f\|_{p}\left(1-x^{2}\right)^{-1 / 4} .
$$

Since $p<4$ this has finite norm.

Returning now to (9.1), we see that Theorem 8.1 is established if we can prove that the functions

$$
(N+1)^{1 / 2}\left(1-x^{2}\right)^{-1 / 4} \int_{-1}^{1} \frac{P_{N}(y)-P_{N+2}(y)}{x-y} f(y) d y
$$

and

$$
(N+1)^{1 / 2}\left(1-x^{2}\right)^{1 / 4} \int_{-1}^{1} \frac{P_{N+1}(y)}{x-y} f(y) d y
$$

are bounded in norm, independently of $N$.

10. The reduction of (9.2). Define the function $A_{n}(y)$ by

$$
A_{n}(y)=(n+1)^{1 / 2}\left(1-y^{2}\right)^{-1 / 4}\left\{P_{n}(y)-P_{n+2}(y)\right\} \text {. }
$$

Then (9.2) is

$$
\begin{aligned}
\int_{-1}^{1} A_{N}(y) & \left\{\left(\frac{1-y^{2}}{1-x^{2}}\right)^{1 / 4}-1+1\right\} \frac{f(y)}{x-y} d y \\
= & \int_{-1}^{1} A_{N}(y)\left\{\frac{\left(\left(1-y^{2}\right) /\left(1-x^{2}\right)\right)^{1 / 4}-1}{x-y}\right\} f(y) d y+\int_{-1}^{1} \frac{A_{N}(y) f(y)}{x-y} d y .
\end{aligned}
$$

Since $A_{N}(y)$ is bounded, by (8.2), the last integral converges almost everywhere as a Cauchy principal value $[10 ; \mathrm{p} .317]$. Then the function defined by (9.2) is in absolute value less than

$$
\begin{aligned}
\int_{-1}^{1}\left|A_{N}(y)\right|\left|\frac{\left(\left(1-y^{2}\right) /\left(1-x^{2}\right)\right)^{1 / 4}-1}{x-y}\right||f(y)| d y & \\
& +\left|\int_{-1}^{1} \frac{A_{N}(y) f(y)}{x-y} d y\right| .
\end{aligned}
$$

For simplicity we write 


$$
K(x, y)=\left|\frac{\left(\left(1-y^{2}\right) /\left(1-x^{2}\right)\right)^{1 / 4}-1}{x-y}\right| .
$$

Then the function (10.1), in view of (8.2), is less than or equal to

$$
A \int_{-1}^{1} K(x, y)|f(y)| d y+\left|\int_{-1}^{1} \frac{A_{N}(y) f(y)}{x-y} d y\right|=A I(x)+J_{N}(x),
$$

say. By a theorem of Riesz [11; p. 317]

$$
\left\|J_{N}(x)\right\|_{p} \leqq A_{p}\left\|A_{N}(y) f(y)\right\|_{p},
$$

and so by (8.2) again

$$
\left\|J_{N}(x)\right\|_{p} \leqq A_{p}\|f(y)\|_{p .}
$$

. It follows that the functions (9.2) are bounded in norm independently of $N$ if we can establish that

$$
I(x)=\int_{-1}^{1} K(x, y)|f(y)| d y
$$

belongs to $L^{p}(-1,1)$.

11. The reduction of (9.3). We now perform a similar reduction for (9.3). Let

$$
B_{n}(y)=(n+1)^{1 / 2}\left(1-y^{2}\right)^{1 / 4} P_{n+1}(y) .
$$

Then $(9.3)$ is

$$
\int_{-1}^{1} B_{N}(y)\left\{\left(\frac{1-x^{2}}{1-y^{2}}\right)^{1 / 4}-1\right\} \frac{f(y)}{x-y} d y+\int_{-1}^{1} \frac{B_{N}(y) f(y)}{x-y} d y .
$$

The $B_{N}(y)$ are uniformly bounded, by (8.1). Arguing as in the preceding section, it follows that the functions (9.3) are bounded in norm independently of $N$ if we can establish that

$$
\int_{-1}^{1} K(y, x)|f(y)| d y
$$

belongs to $L^{p}(-1,1)$.

12. An inequality. In the two preceding sections we have reduced the proof of Theorem 8.1 to the following lemma.

Lemma 12.1. If $f(x) \in L^{p}(-1,1), 4 / 3<p<4$, then so do

$$
\int_{-1}^{1} K(x, y) f(y) d y
$$

and 


$$
\int_{-1}^{1} K(y, x) f(y) d y .
$$

$K(x, y)$ is defined as in (10.2).

By the converse of Hölder's inequality it is enough to show that for every function $g(x)$ in $L^{p^{\prime}}(-1,1), p^{\prime}=p /(p-1)$, the integrals

$$
\begin{aligned}
& \int_{-1}^{1} g(x) d x \int_{-1}^{1} K(x, y) f(y) d y \\
& \int_{-1}^{1} g(x) d x \int_{-1}^{1} K(y, x) f(y) d y
\end{aligned}
$$

converge. We may assume that $g(x) \geqq 0, f(x) \geqq 0$. It suffices to prove the convergence of one of these iterated integrals, for inverting the order of integration and interchanging the rules of $p$ and $p^{\prime}$ turns one case into the other. We therefore confine our attention to (12.1).

The iterated integral (12.1) can be written

$$
\int_{-1}^{1} \int_{-1}^{1} f(y) K^{1 / p}(x, y)\left(\frac{1-y^{2}}{1-x^{2}}\right)^{1 / p p^{\prime}} \cdot g(x) K^{1 / p^{\prime}}(x, y)\left(\frac{1-y^{2}}{1-x^{2}}\right)^{-1 / p p^{\prime}} d x d y .
$$

By Hölder's inequality for double integrals, this is less than or equal to

$$
\begin{aligned}
\left\{\int_{-1}^{1} g(x)^{p^{\prime}} d x \int_{-1}^{1} K(x, y)\right. & \left.\left(\frac{1-y^{2}}{1-x^{2}}\right)^{-1 / p} d y\right\}^{1 / p^{\prime}} \\
& \cdot\left\{\int_{-1}^{1} f(y)^{p} d y \int_{-1}^{1} K(x, y)\left(\frac{1-y^{2}}{1-x^{2}}\right)^{1 / p^{\prime}} d x\right\}^{1 / p} .
\end{aligned}
$$

In view of the fact that $f \in L^{p}$ and $g \in L^{p^{\prime}}$ the convergence of (12.1) will be established if the functions

$$
\begin{aligned}
& \int_{-1}^{1} K(x, y)\left(\frac{1-y^{2}}{1-x^{2}}\right)^{-1 / p} d y \\
& \int_{-1}^{1} K(x, y)\left(\frac{1-y^{2}}{1-x^{2}}\right)^{1 / p^{\prime}} d x
\end{aligned}
$$

are essentially bounded in $-1 \leqq x \leqq 1$ and $-1 \leqq y \leqq 1$ respectively. By the definition (10.2) of $K(x, y)$ both these functions are of the form

$$
\int_{-1}^{1}|s-t|^{-1}\left|\left(\frac{1-t^{2}}{1-s^{2}}\right)^{a}-\left(\frac{1-t^{2}}{1-s^{2}}\right)^{b}\right| d s,
$$

where $0<a<1,0<b<1,-1<t<1$. We shall show in the next section that this function of $t$ is bounded. 
Before doing so we pause to point out that our proof of Lemma 12.1 implies the interesting inequality

$$
\left\|\int_{-1}^{1} K(x, y) f(y) d y\right\|_{p} \leqq A\|f\|_{p}
$$

for $4 / 3<p<4$. A generalization of this has been stated by the author without proof in the note [5].

13. The proof completed. The function (12.2) can be written in the form

$$
\int_{-1}^{1}\left|\frac{(\cdots)^{a}-(\cdots)^{b}}{s^{2}-t^{2}}\right||s+t| d s,
$$

where the meaning of the dots is clear. This is less than or equal to

$$
\int_{-1}^{1}\left|\frac{(\cdots)^{a}-(\cdots)^{b}}{s^{2}-t^{2}}\right||s| d s+|t| \int_{-1}^{1}\left|\frac{(\cdots)^{a}-(\cdots)^{b}}{s^{2}-t^{2}}\right| d s .
$$

In view of the fact that each integrand is even we may replace the integrals $\int_{-1}^{1}$ by $2 \int_{0}^{1}$. Now let $u=1-t^{2}, v=1-s^{2}$, and the last two expressions become, apart from multiplicative constants,

$$
\begin{gathered}
\int_{0}^{1}\left|\frac{(u / v)^{a}-(u / v)^{b}}{u-v}\right| d v, \\
(1-u)^{1 / 2} \int_{0}^{1}\left|\frac{(u / v)^{a}-(u / v)^{b}}{u-v}\right| \frac{d v}{(1-v)^{1 / 2}}
\end{gathered}
$$

respectively. Each of these is to be shown bounded in $0<u<1$.

(13.1) can be disposed of by making the change of variable $v=\lambda u$. It becomes

$$
\int_{0}^{1 / u}\left|\frac{\lambda^{-a}-\lambda^{-b}}{1-\lambda}\right| d \lambda
$$

which is bounded by

$$
\int_{0}^{\infty}\left|\frac{\lambda^{-a}-\lambda^{-b}}{1-\lambda}\right| d \lambda
$$

This converges since $0<a<1,0<b<1$.

(13.2) is a trifle more delicate. Again let $v=\lambda u$, and it becomes

$$
\begin{aligned}
(1-u)^{1 / 2}\left(\int_{0}^{1}+\int_{1}^{1 / u}\right. & \left|\frac{\lambda^{-a}-\lambda^{-b}}{1-\lambda}\right| \frac{d \lambda}{(1-\lambda u)^{1 / 2}} \\
& \leqq \int_{0}^{1}\left|\frac{\lambda^{-a}-\lambda^{-b}}{1-\lambda}\right| d \lambda+\int_{1}^{1 / u}\left|\frac{\lambda^{-a}-\lambda^{-b}}{1-\lambda}\right| \frac{d \lambda}{(1-\lambda u)^{1 / 2}} .
\end{aligned}
$$


The last term is less than or equal to

$$
A \int_{1}^{1 / u} \lambda^{-1-c}(1-\lambda u)^{-1 / 2} d \lambda
$$

where $c=\min (a, b)>0$. Reverting to the original variable $v=\lambda u$ turns the integral into

$$
u^{c} \int_{u}^{1} v^{-1-c}(1-v)^{-1 / 2} d v
$$

This approaches the limit $1 / c$ as $u \rightarrow 0$, and so must be bounded for $0 \leqq u \leqq 1$.

Theorem 8.1 stands established.

Chapter III. ORThogonal SETS OF SPHERICAL haRMonics

14. Properties of spherical harmonics. In this brief survey we follow Hille's dissertation [7], where further information can be found. The roots of the equation $\left({ }^{6}\right)$

$$
\psi(\alpha)+\psi(-1-\alpha)=K,
$$

when $K<2 \psi(-1 / 2)$, are all real, different from $\alpha=-1 / 2$, and are symmetric in this point. Denoting those on the right of the point by $\alpha_{k}, k=0,1, \cdots$, we find that $-1 / 2<\alpha_{0}<0, n<\alpha_{n}<n+1, n>0$. The functions $\left\{P_{\alpha_{n}}(x)\right\}_{0}^{\infty}$ are orthogonal and closed $L^{2}(-1,1)[7 ;$ p. 56]. Boas and Pollard [13] have shown that they are in fact closed $L^{p}(-1,1)$ for all $p>1$. To normalize the functions we use the formula $[7 ; 56]$

$$
\sigma_{n}^{2}=\int_{-1}^{1} P_{\alpha_{n}}^{2}(x) d x=\frac{2 \sin ^{2} \alpha_{n} \pi}{\pi^{2}\left(2 \alpha_{n}+1\right)}\left\{\psi^{\prime}\left(-1-\alpha_{n}\right)-\psi^{\prime}\left(\alpha_{n}\right)\right\}
$$

The formal expansion of any function $f(x) \in L^{p}(-1,1), p>1$, takes the form

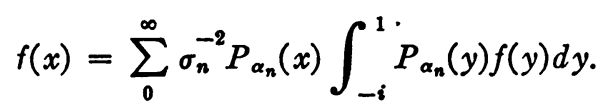

We note in passing that the $\left\{\alpha_{n}\right\}$ reduce to the integers $\{n\}$ if $K=-\infty$.

TheOREM 14.1. The functions $\left\{P_{\alpha_{n}}(x)\right\}$ defined above form a basis for $L^{p}(-1,1)$ if $4 / 3<p<4$, but not if $1<p<4 / 3$ or $p>4$.

The proof will follow the lines of Chapter I.

15. A contour integral. Suppose $-1<s<1,-1<t<1, \alpha_{N+1}>R_{N}>N$. Define a contour $C(N)$ as follows. It consists of the line segment joining $-1 / 2$ $+i R_{N}$ to $-1 / 2-i R_{N}$, closed on the right by the semicircular arc $C_{1}(N)$ with

$(0) \psi(x)$ is defined as $\Gamma^{\prime}(x+1) / \Gamma(x+1)$. 
this segment as diameter. Let

$$
I=\frac{1}{2 \pi i} \int_{C(N)} \frac{\pi^{2}(2 z+1) P_{z}(s) P_{z}(t) d z}{2 \sin ^{2} \pi z[\psi(-1-z)+\psi(z)-K]} .
$$

In view of equation (14.1) the integrand has simple poles $\alpha_{0}, \alpha_{1}, \cdots, \alpha_{N}$ and $0,1, \cdots, N$. Hence, by the residue theorem

$$
\text { (15.1) } I=\sum_{n=0}^{N}(n+1 / 2) P_{n}(s) P_{n}(t)-\sum_{n=0}^{N} \frac{\pi^{2}\left(2 \alpha_{n}+1\right) P_{\alpha_{n}}(s) P_{\alpha_{n}}(t)}{2 \sin ^{2} \pi \alpha_{n}\left[\psi^{\prime}\left(\alpha_{n}\right)-\psi^{\prime}\left(-1-\alpha_{n}\right)\right]} .
$$

The integral along the line segment vanishes, for it is equal to

$$
\text { const. } \int_{R_{N}}^{-R_{N}} \frac{y P_{-1 / 2+i y}(s) P_{-1 / 2+i y}(t) d y}{\cosh ^{2} y\{\psi(-1 / 2+i y)+\psi(-1 / 2-i y)-K\}}
$$

which has an odd integrand $\left({ }^{7}\right)$. Then

$$
I=\frac{\pi}{4 i} \int_{C_{1}(N)} \frac{(2 z+1) P_{z}(s) P_{z}(t) d z}{2 \sin ^{2} \pi z\{\psi(-1-z)+\psi(z)-K\}} .
$$

16. Estimate of the integral $I$. According to the formula [12; p. 30]

$$
P_{z}(\cos \theta)=\frac{2^{1 / 2}}{\pi} \int_{0}^{\theta} \frac{\cos (z+1 / 2) \psi}{(\cos \psi-\cos \theta)^{1 / 2}}, \quad . z=x+i y, 0<\theta<\pi,
$$

we have

$$
\begin{aligned}
\left|P_{z}(\cos \theta)\right| & \leqq A e^{\theta|y|} \int_{0}^{\theta} \frac{d \psi}{(\cos \psi-\cos \theta)^{1 / 2}} \\
& \leqq A e^{\theta|y| P_{-1 / 2}(\cos \theta) .}
\end{aligned}
$$

By an asymptotic formula of Hille $[7 ;$ p. 6] this is in turn less than or equal to

$$
A e^{\theta|y|} \log (\pi-\theta) \text {. }
$$

Now let $s=\cos \theta, t=\cos \phi$. Then the integrand of $I$ is (cf. [7; p. 62]) less than or equal to

$$
A e^{-(2 \pi-\theta-\phi)|y|} \log (\pi-\theta) \log (\pi-\phi)
$$

so

$$
\begin{aligned}
|I| & \leqq A \log (\pi-\theta) \log (\pi-\phi) \int_{C_{1}(N)} e^{-(2 \pi-\theta-\phi)|y|}|d z| \\
& \leqq A \log (\pi-\theta) \log (\pi-\phi)(2 \pi-\theta-\phi)^{-1},
\end{aligned}
$$

by the argument used in $\$ 5$. From (14.2) and (15.1) it follows then that

( $)$ We have used here the fact that $P_{-1 / 2-i y}(s)=P_{-1 / 2+i y}(s)$. 


$$
\left|\sum_{.0}^{N}(n+1 / 2) P_{n}(s) P_{n}(t)-\sum_{0}^{N} \sigma_{n}^{-2} P_{\alpha_{n}}(s) P_{\alpha_{n}}(t)\right| \leqq A T(s, t),
$$

where

$$
T(s, t)=\frac{\log (\pi-\arccos s) \log (\pi-\arccos t)}{2 \pi-\arccos s-\arccos t} .
$$

LeMma 16.1. If $f(s) \in L^{p}(-1,1), g(t) \in L^{p^{\prime}}(-1,1), p>1$, then

$$
\int_{-1}^{1} g(t) d t \int_{-1}^{1} T(s, t) f(s) d s
$$

converges.

On a change of variables the iterated integral becomes

$$
\begin{aligned}
\int_{0}^{\pi} \log (\pi-\phi) g(\cos \phi) \sin \phi d \phi \int_{0}^{\pi} \frac{\log (\pi-\theta) f(\cos \theta) \sin \theta}{2 \pi-\theta-\phi} d \theta \\
=\int_{0}^{\pi} \log \phi g(-\cos \phi) \sin \phi d \phi \int_{0}^{\pi} \frac{\log \theta f(-\cos \theta) \sin \theta}{\theta+\phi} d \theta .
\end{aligned}
$$

It is easily verified that

$$
\begin{aligned}
& \log \phi g(-\cos \phi) \sin \phi \in L^{p^{\prime}}(0, \pi), \\
& \log \theta f(-\cos \theta) \sin \theta \in L^{p}(0, \pi) .
\end{aligned}
$$

Then the convergence of the integral follows from Hilbert's inequality [6; 226].

Corollary 16.1. If $f(s) \in L^{p}(-1,1), p>1$, so does

$$
\int_{-1}^{1} T(s, t) f(s) d s
$$

17. Proof of Theorem 14.1. Suppose $f(s) \in L^{p}(-1,1), p>1$. Let $S_{N}(f)$ denote the partial sums of its formal expansion (14.3), and $\sum_{N}(f)$ the partial sums of its Legendre series. By (16.1) and the corollary just established $\left\|\sum_{N}(f)-S_{N}(f)\right\|_{p}$ is bounded in $N$.

If $4 / 3<p<4, \lim \sup _{N \rightarrow \infty}\left\|\sum_{N}(f)\right\|_{p}$ is finite, by Theorem 8.1 . Hence the same is true of lim $\sup _{N \rightarrow \infty}\left\|S_{N}(f)\right\|$. This proves the first part of Theorem 14.1.

Now suppose $4 / 3<p<4$, or $p>4$. Since the Legendre polynomials are not a basis for this range of $p$, there is a function $f(s)$ such that $\lim \sup _{N \rightarrow \infty}\left\|\sum_{N}(f)\right\|_{p}=\infty$. It follows then that lim $\sup _{N \rightarrow \infty}\left\|S_{N}(f)\right\|_{p .}$ This proves the second part of Theorem 14.1. 


\section{REFERENCES}

1. M. Riesz, Sur les fonctions conjugu\&es, Math. Zeit. vol. 27 (1927) pp. 218-244.

2. J. Schauder, Eine Eigenschaft der Haarschen Orthogonal-system, Math. Zeit. vol. 28 (1928) pp. 317-320.

3. H. Kober, $A$ note on approximation by rational functions, Proceedings of the Edinburgh Mathematical Society vol. 7 (1946) pp. 123-133.

4. W. B. Caton and E. Hille, Laguerre polynomials and Laplace integrals, Duke Math. J. vol. 12 (1945) pp. 217-242.

5. H. Pollard, The mean convergence of orthogonal series of polynomials, Proc. Nat. Acad. Sci. U. S. A. vol. 32 (1946) pp. 8-10.

6. G. H. Hardy, J. E. Littlewood, and G. P6lya, Inequalities, Cambridge, 1933.

7. E. Hille, Some problems concerning spherical harmonics, Arkiv för Matematik, Astronomi och Fysik vol. 13 (1918) pp. 1-76.

8. S. Banach, Theorie des operations lineaires, Warsaw, 1932.

9. N. Levinson, Gap and density theorems, Amer. Math. Soc. Colloquium Publications, vol. 26, New York, 1940.

10. A. Zygmund, Trigonometrical series, Warsaw, 1935.

11. G. Szegö, Orthogonal polynomials, Amer. Math. Soc. Colloquium Publications, vol. 23, New York, 1939.

12. E. W. Watson, The theory of spherical and ellipsoidal harmonics, Cambridge, 1931.

13. R. P. Boas and H. Pollard, Complete sets of Bessel and Legendre functions, Ann. of Math. vol. 48 (1947) pp. 366-384.

14. R. E. A. C. Paley, A remarkable system of orthogonal functions, Proc. London Math. Soc. vol. 34 (1932) pp. 241-279.

\section{CORNELl UNIVERSity,}

ITHACA, N. Y. 